\title{
Increased blood group 2 innate lymphoid cells are involved in blood eosinophilia and itching in Kimura disease
}

\author{
Ichiro Tojima ${ }^{1}$, Takuya Murao ${ }^{1}$, Keigo Nakamura ${ }^{1}$, Hiroyuki Arai ${ }^{1}$, Shino Shimizu ${ }^{1}$, \\ Hideaki Kouzaki ${ }^{1}$, and Takeshi Shimizu ${ }^{1}$ \\ ${ }^{1}$ Shiga Ika Daigaku Igakubu
}

January 10, 2022

\begin{abstract}
Background: Kimura disease (KD) is a rare, chronic inflammatory disorder characterized by blood eosinophilia, general itching, and subcutaneous head and neck mass lesions; however, the etiology of this disease is unknown. We hypothesized that group 2 innate lymphoid cells (ILC2s) in peripheral blood may play an essential role in the pathogenesis of KD. Methods: The prevalence of blood ILC2s and their ability to produce interleukin (IL) -4, IL-5, IL-13, and IL-31 in patients with KD were compared with those in control subjects and in patients with house dust mite (HDM) -induced allergic rhinitis (AR). Changes in blood ILC2 prevalence, blood eosinophilia, and clinical symptoms after surgery and steroid therapy were evaluated. Results: Blood ILC2 prevalence in patients with KD were eight times and six times higher than those in control subjects and in patients with AR, respectively. There was a strong positive correlation between ILC2 prevalence and blood eosinophilia. Patients with KD showed increased serum IL-13 and decreased IL-31 levels. KD patient-derived blood ILC2s produced large amounts of IL-5 and IL-13 in response to prostaglandin (PG) $\mathrm{D}_{2}$ and leukotriene (LT) $\mathrm{D}_{4}$, compared to ILC2s derived from control subjects and patients with AR. Surgery and systemic steroid therapy ameliorated general itching with a concomitant decrease in blood ILC2s and blood eosinophilia. Upon disease recurrence, blood ILC2 prevalence and blood eosinophilia increased concurrently with general itching. Conclusion: Increased blood ILC2s may be involved in blood eosinophilia and general itching through the production of IL-5 and IL-13 in patients with KD.
\end{abstract}

Original article

Title:

Increased blood group 2 innate lymphoid cells are involved in blood eosinophilia and itching in Kimura disease

Short title:

Increased blood ILC2s in Kimura disease

Ichiro Tojima, MD, PhD*, Takuya Murao, MD, Keigo Nakamura, MD, Hiroyuki Arai, MD, Shino Shimizu, MD, PhD, Hideaki Kouzaki, MD, PhD, Takeshi Shimizu, MD, PhD

Department of Otorhinolaryngology-Head and Neck Surgery, Shiga University of Medical Science, Otsu, Japan

*Corresponding Author: Ichiro Tojima, MD, PhD, Department of Otorhinolaryngology, Shiga University of Medical Science, Seta-Tsukinowa, Otsu, Shiga 520-2192, Japan; Tel: +81-77-548-2261; Fax: +81-77-5482783; E-mail: itirotz@belle.shiga-med.ac.jp

Funding Statement: 
This research was supported, in part, by the Japan Society for the Promotion of Science (JSPS; Grant Numbers 17K16908 and 20K09754).

Disclosure Statement:

The authors declare that they have no relevant conflicts of interest.

\section{Acknowledgments}

We thank the staff of the central research laboratory of the Shiga University of Medical Science for their important contributions to the completion of this work.

\section{Abstract}

Background: Kimura disease (KD) is a rare, chronic inflammatory disorder characterized by blood eosinophilia, general itching, and subcutaneous head and neck mass lesions; however, the etiology of this disease is unknown. We hypothesized that group 2 innate lymphoid cells (ILC2s) in peripheral blood may play an essential role in the pathogenesis of KD.

Methods: The prevalence of blood ILC2s and their ability to produce interleukin (IL) -4, IL-5, IL-13, and IL-31 in patients with KD were compared with those in control subjects and in patients with house dust mite (HDM) -induced allergic rhinitis (AR). Changes in blood ILC2 prevalence, blood eosinophilia, and clinical symptoms after surgery and steroid therapy were evaluated.

Results: Blood ILC2 prevalence in patients with KD were eight times and six times higher than those in control subjects and in patients with AR, respectively. There was a strong positive correlation between ILC2 prevalence and blood eosinophilia. Patients with KD showed increased serum IL-13 and decreased IL-31 levels. KD patient-derived blood ILC2s produced large amounts of IL-5 and IL-13 in response to prostaglandin (PG) $\mathrm{D}_{2}$ and leukotriene (LT) $\mathrm{D}_{4}$, compared to ILC2s derived from control subjects and patients with AR. Surgery and systemic steroid therapy ameliorated general itching with a concomitant decrease in blood ILC2s and blood eosinophilia. Upon disease recurrence, blood ILC2 prevalence and blood eosinophilia increased concurrently with general itching.

Conclusion: Increased blood ILC2s may be involved in blood eosinophilia and general itching through the production of IL-5 and IL-13 in patients with KD.

\section{Key words}

eosinophil, group 2 innate lymphoid cells, IL-13, itching, Kimura disease

\section{Abbreviations used}

AR: Allergic rhinitis; ILC2: Group 2 innate lymphoid cell; HDM: House dust mite; IL-4R $\alpha$ : IL-4 receptor subunit- $\alpha$ KD: Kimura disease; LT: Leukotriene; PBMC: Peripheral blood mononuclear cell; PG: Prostaglandin; PSL: Prednisolone; VAS: Visual analog scale

\section{Main text}

\section{Introduction}

Kimura disease (KD) is a chronic inflammatory disorder characterized by general itching and subcutaneous head and neck mass lesions with tissue and blood eosinophilia. ${ }^{1} \mathrm{KD}$ is a rare disorder, and 238 cases of $\mathrm{KD}$ have been reported worldwide in the last 30 years. $^{2}$ This disease is found predominantly in young men in Asian countries. Although the mass lesions may be eradicated by surgery and steroid therapy, the disease frequently recurs. Laboratory tests show marked blood eosinophilia and elevated serum IgElevels. However, the precise etiology of this disease is unknown. Several researchers have reported the role of type 2 cytokines in the pathogenesis of KD. Interleukin (IL) -4- and IL-5-expressing mast cells and T cells accumulate in local lesions, ${ }^{3}$ and the expression of mRNAs encoding IL-4, IL-5, and IL-13 in peripheral blood mononuclear cells (PBMCs) was shown to correlate with the number of blood eosinophils in patients with KD. ${ }^{4}$ 
Itching is the most frequent symptom in KD. ${ }^{2}$ IL-4 and IL-13 directly stimulate sensory neurons via IL-4 receptor subunit- $\alpha(\mathrm{IL}-4 \mathrm{R} \alpha),{ }^{5}$ and consequently, these cytokines are more critical than histamine for itching. ${ }^{6}$ IL-31, produced primarily by Th2 cells, ${ }^{7,8}$ also acts directly on itch-sensory neurons to induce itching. ${ }^{5}$ There is a positive correlation between serum IL-31 levels and the severity of atopic dermatitis, ${ }^{8}$ and an anti-IL-31 receptor $\alpha$ antibody has been shown to ameliorate itching in patients with atopic dermatitis. ${ }^{6}$

Group 2 innate lymphoid cells (ILC2s) have been identified as important effector cells for eosinophilic inflammatory processes such as allergic rhinitis (AR), chronic rhinosinusitis, asthma, and atopic dermatitis. ${ }^{9}$ ILC2s play critical roles in the induction of type 2 inflammation through the production of IL-4, IL-5, and IL-13 in response to various mediators such as IL-33, prostaglandin (PG) $\mathrm{D}_{2}$, and leukotriene (LT) $\mathrm{C}_{4}, \mathrm{D}_{4}$, and $\mathrm{E}_{4} \cdot{ }^{10,11} \mathrm{PGD}_{2}$ and these LTs are synthesized by eosinophils and mast cells ${ }^{12,13}$ in response to several factors, including IL-5 and IL-13. Our previous study revealed that ILC2s in nasal turbinate tissues of patients with house dust mite (HDM) -induced AR and those in nasal polyp tissues of patients with eosinophilic chronic rhinosinusitis were increased, an effect that correlated positively with the number of infiltrating eosinophils.

Kimura disease is characterized by blood eosinophilia, high serum IgE levels, and itching symptoms. We hypothesized that blood ILC2s may play an essential role in the pathogenesis of KD through the production of type 2 cytokines. In the present study, we examined the prevalence of blood ILC2s; their ability to produce IL-4, IL-5, IL-13, and IL-31; and serum concentrations of these type 2 cytokines in patients with $\mathrm{KD}$, compared with those in control subjects and in patients with HDM-induced AR. We also showed the role of blood ILC2s in blood eosinophilia and itching symptoms by presenting a severe case of KD, followed for more than 6 years, that was treated with surgery and systemic steroid therapy.

\section{Methods}

\subsection{Study subjects}

A total of four patients with KD (4 males), 16 patients with HDM-induced AR (11 males and 5 females), and 13 control subjects ( 8 males and 5 females) were recruited from the Department of Otorhinolaryngology at the Shiga University of Medical Science hospital according to protocols approved by the Institutional Review Board of the Shiga University of Medical Science (Ethics Approval Numbers 28-106 and R2019-248). Informed consent was obtained from all participants. The patients with HDM-induced AR had a history of perennial AR and were diagnosed based on the results of the nasal provocation test, skin prick test, and serum-specific IgE levels ([?]0.7 UA/mL), according to the criteria established by Bousquet et al. ${ }^{14}$ Asthmatic subjects, patients with chronic rhinosinusitis, or current users of oral steroids were excluded. The clinical data for the study subjects are listed in Table 1.

\subsection{Visual analog scale (VAS) for KD}

VAS provides quantitative measures that have been validated for multiple diseases, and was applied herein to assess the severity of KD as well as the efficacy of therapeutic interventions. The VAS scale ranges from 0 (absence of symptoms) to 10 (very severe symptoms) for general itching and distress. The general distress score is an overall score that includes frustration, reduced productivity, reduced concentration, and lack of a good night's sleep.

\subsection{Isolation of ILC2s from peripheral blood}

PBMCs were isolated from control subjects, patients with HDM-induced AR, or patients with KD using Histopaque 1083 (Sigma-Aldrich, St. Louis, MO). Cells were treated (30 minutes, $4{ }^{\circ} \mathrm{C}$, in the dark) with Red LIVE/DEAD fixable dead cell-staining reagent (Invitrogen, Carlsbad, CA) as a live/dead discriminator, and then washed. Cells were blocked $\left(10\right.$ minutes, $4{ }^{\circ} \mathrm{C}$, in the dark) with Fc Blocking Reagent (Miltenyi Biotec, Auburn, CA). Cells then were incubated $\left(30\right.$ minutes, $4{ }^{\circ} \mathrm{C}$, in the dark) with the following antibodies: fluorescein isothiocyanate (FITC) -conjugated anti-lineage cocktail (anti-CD3, -CD14, -CD16, -CD19, -CD20, and-CD56), FITC-conjugated anti-CD11b, FITC-conjugated anti-CD11c, FITC-conjugated anti-CD123, FITC-conjugated anti-TCR $\alpha \beta$, FITC-conjugated anti-TCR $\gamma \delta$, FITC-conjugated anti-FceR1 $\alpha$, phycoerythrin (PE) -conjugated anti-CD127, PE-cyanin 7 (PE-Cy7) -conjugated anti-CD45, and Alexa Fluor 
647-conjugated anti-chemoattractant receptor-homologous molecule-expressed on Th2 cells (CRTH2). All antibodies were purchased from BioLegend (San Diego, CA) or eBioscience (San Diego, CA). Lineage (CD3, CD11b, CD11c, CD14, CD16, CD19, CD20, CD56, CD123, TCR $\alpha \beta$, TCR $\gamma \delta$, and FceR1 $\alpha$ ) -negative, CD45 ${ }^{+}$, $\mathrm{CD} 127^{+}, \mathrm{CRTH}_{2}{ }^{+}$cells were identified as ILC2s (Figure S1) using a FACSAria (BD Biosciences, San Jose, CA). All analysis was performed with FlowJo software, version 9.3 (TreeStar, Ashland, OR). The prevalence of blood ILC2s was calculated as the number of ILC2s divided by the number of total CD $45^{+}$cells. The purity of blood ILC2s was greater than $98 \%$ (data not shown).

\subsection{Production of IL-4, IL-5, IL-13, and IL-31 from blood ILC2s}

Sorted ILC2s from PBMCs were incubated (500 cells/well) in 96-well round-bottom plates for 7 days at 37 ${ }^{\circ} \mathrm{C}$ in RPMI 1640 containing $10 \%$ autologous serum, penicillin (100 U/mL, Wako, Osaka, Japan), streptomycin (100 $\mu \mathrm{g} / \mathrm{mL}$, Wako), and IL-2 (50 ng/mL; PeproTech) with or without IL-33 (10 ng/mL; PeproTech, Rocky Hill, NJ), $\mathrm{PGD}_{2}\left(1 \mu \mathrm{M}\right.$; Cayman Chemical, Ann Arbor, MI), or $\mathrm{LTD}_{4}$ (50 nM; Cayman Chemical). These concentrations of IL-33, $\mathrm{PGD}_{2}$, and $\mathrm{LTD}_{4}$ were determined as previously described. ${ }^{10}$ In all in vitro experiments, ILC2s were incubated with IL-2 to promote the proliferation and cell survival of ILC2s. ${ }^{11}$

For the in vitro experiments using dexamethasone, PBMC-derived ILC2s proliferated as described previously. ${ }^{10}$ Briefly, sorted ILC2s from patients with KD have incubated in 96 -well round-bottom plates for 3 weeks. Half of the medium was refreshed every 2 to 3 days. Then, proliferated ILC2s were stimulated with or without $\mathrm{PGD}_{2}(1 \mu \mathrm{M})$ or $\mathrm{LTD}_{4}(50 \mathrm{nM})$, with or without dexamethasone $\left(10^{-8}\right.$ to $\left.10^{-6} \mathrm{M}\right)$ for $12 \mathrm{~h}$.

The concentrations of IL-4, IL-5, IL-13, and IL-31 in cell-free supernatants were determined using ELISA kits (R\&D Systems, Minneapolis, MN), according to the manufacturer's instructions.

\subsection{Serum concentrations of IL-4, IL-5, IL-13, and IL-31}

Serum concentrations of IL-4, IL-5, IL-13, and IL-31 were measured using ELISA kits (RayBiotech, Inc., Norcross, GA, for IL-4, IL-5, and IL-13; R\&D Systems for IL-31), according to the respective manufacturer's instructions. The detection limits of the assays were as follows: IL-4, $5.0 \mathrm{pg} / \mathrm{mL}$; IL-5, $5.0 \mathrm{pg} / \mathrm{mL}$; IL-13, $0.15 \mathrm{pg} / \mathrm{mL}$; and IL-31, $31.3 \mathrm{pg} / \mathrm{mL})$.

\subsection{Statistical analysis}

Data are presented as mean \pm SEM for the number of experiments indicated. Differences between groups were analyzed using the two-tailed Kruskal-Wallis test with Dunn's post hoc test or two-tailed Mann-Whitney $U$ test. Correlations were assessed using Spearman's rank correlation. $P$ values of less than 0.05 were considered statistically significant. All statistical analyses were performed with IBM SPSS Statistics 25 (SPSS, Inc., Tokyo, Japan).

\section{Results}

\subsection{Prevalence of blood ILC2s}

Clinical data for the study subjects are listed in Table 1. None of the patients with KD had atopic dermatitis, asthma, or AR. The prevalence of blood ILC2s in patients with KD (mean \pm SEM, $2.35 \pm 0.90 \%$ of CD45 cells) was eight times and six times higher than those in control subjects $(0.29 \pm 0.03 \%)$ and in patients with AR $(0.37 \pm 0.04 \%$, Figure $1 \mathrm{~A}$ and $\mathrm{B})$, respectively. Correlation analysis in all study subjects revealed a strong positive correlation between the prevalence of ILC2s and percentage of eosinophils in blood $(\mathrm{R}=$ $0.78, P<0.001$, Figure $1 \mathrm{C})$. There was a weak positive correlation between blood ILC2 prevalence and serum IgE levels $(\mathrm{R}=0.36, P<0.05$, Figure 1D).

\subsection{Serum concentrations of IL-4, IL-5, IL-13, and IL-31}

Serum IL-13 levels were significantly elevated in patients with KD compared to those in patients with AR and control subjects (Figure 2A). Serum IL-5 levels also were elevated, although this effect fell short of statistical insignificance, while IL-4 levels were not apparently changed (Figure 2B and C). Serum IL-31 levels were significantly decreased in patients with KD compared to those in patients with AR and in control 
subjects (Figure 2D). There was a weak positive correlation between blood ILC2 prevalence and serum IL-13 levels $(\mathrm{R}=0.35, P=0.09$, Figure $3 \mathrm{~A})$. However, there was no correlation between blood ILC2 prevalence and serum levels of IL-5, IL-4, or IL-31 (Figure 3B-D).

\subsection{Production of IL-4, IL-5, IL-13, and IL-31 from blood ILC2s}

To examine the ability of blood ILC2s to produce IL-4, IL-5, IL-13, and IL-31, ILC2s were incubated (starting immediately after sorting) with IL-33 $(10 \mathrm{ng} / \mathrm{mL}), \mathrm{PGD}_{2}(1 \mu \mathrm{M})$, or $\mathrm{LTD}_{4}(50 \mathrm{nM})$ for 7 days. $\mathrm{PGD}_{2}$ or $\mathrm{LTD}_{4}$ stimulated the production of IL-4, IL-5, and IL-13 by ILC2s derived from patients with KD (Figure 4A); the production of IL-5 and IL-13 was significantly increased when compared with that by ILC2s from patients with AR and control subjects (Figure 4B). Sorted blood ILC2s did not produce detectable levels of IL-31.

To examine the effects of corticosteroid on ILC2-induced type 2 cytokine productions, proliferated PBMCderived ILC2s from patients with KD were incubated with or without $\mathrm{PGD}_{2}(1 \mu \mathrm{M})$ or $\mathrm{LTD}_{4}(50 \mathrm{nM})$ for $12 \mathrm{~h}$. $\mathrm{PGD}_{2}$-induced and $\mathrm{LTD}_{4}$-induced IL-5 and IL-13 productions were significantly inhibited by dexamethasone dose-dependently (Figure 4C).

\subsection{Impact of surgery and systemic steroid therapy on blood ILC2s in a case of KD}

We present the representative severe case of a 41-year-old man with KD. This patient was referred to our hospital complaining of a 7-year history of general itching and swelling on his right upper neck. A $7.0 \mathrm{~cm} \mathrm{x}$ $5.8 \mathrm{~cm}$ hard mass was found in his right parotid area. Laboratory data revealed blood eosinophilia (15.9\% of total leukocytes) and high serum IgE level $(1434 \mathrm{IU} / \mathrm{mL})$. His blood ILC2 prevalence was elevated $(4.9 \%$ of $\mathrm{CD} 45^{+}$cells).

The VAS scores of general itching (10/10) and distress (10/10) were very severe, and a right superficial parotidectomy was performed. Histological examination revealed the characteristic features of KD; lymphoid hyperplasia with reactive germinal centers and massive eosinophil infiltrations (380 cells/high-power field) with microabscess formation. After surgery, we started the patient on $125 \mathrm{mg} /$ day $(2 \mathrm{mg} / \mathrm{kg}$ ) prednisolone (PSL) with subsequent tapering for one month, and the postoperative course was uneventful. The prevalence of blood ILC2s (1.1\%) and blood eosinophils (0.1\%) were decreased one day after surgery (Figure 5A). These parameters remained low for one month during steroid therapy, and clinical symptoms (general itching and distress) were eliminated completely (Figure 5A and B).

Three years after surgery, the patient again complained of general itching and swelling on his right parotid area. Five years after surgery, the VAS scores were worsened (general itching: 9/10, distress: 8/10), and the patient again exhibited high blood ILC2 prevalence (5.4\%), blood eosinophilia (17\%), high serum IgE level $(1009 \mathrm{IU} / \mathrm{mL})$, and parotid swelling (Figure 5A-D). Oral PSL treatment (30 mg/day, $0.5 \mathrm{mg} / \mathrm{kg}$ ) with tapering for one month ameliorated the parotid swelling and the VAS scores (general itching: 1.5/10, distress: 3/10). Blood ILC2 prevalence (1.6\%), blood eosinophilia (11.7\%), and serum IgE level (509.5 IU/mL) also were decreased (Figure 5A, B, and D). After stopping the PSL treatment, blood ILC2 prevalence, blood eosinophilia, and the VAS scores gradually increased again.

\section{4 discussion}

This work is the first report (to our knowledge) showing the prevalence of blood ILC2s and their increased ability to produce IL-5 and IL-13 in patients with KD. The prevalence of blood ILC2s in patients with $\mathrm{AR}$ has been reported to be higher than that in control subjects, ${ }^{15.16}$ and a positive correlation has been demonstrated between the prevalence of blood ILC2s and clinical symptoms of AR. ${ }^{15}$ We previously showed increased IL-5 production from AR patient-derived blood ILC2s in response to $\mathrm{PGD}_{2}$ or $\mathrm{LTD}_{4} \cdot{ }^{10}$ In the present study, the prevalence of blood ILC2s in patients with KD was six times higher than that in patients with AR. Serum concentrations of IL- 5 and IL-13 were elevated in patients with KD, and KD patient-derived blood ILC2s stimulated with $\mathrm{PGD}_{2}$ or $\mathrm{LTD}_{4}$ produced increased amounts of IL-5 and IL-13, compared to ILC2s derived from control subjects or from patients with AR. Additionally, blood ILC2 prevalence correlated positively with serum concentrations of IL-13. These results suggest that an increased number of blood ILC2s 
and their increased ability to produce IL-5 and IL-13 are involved in the elevated serum levels of IL-5 and IL-13 in patients with KD.

It is well-known that ILC2s in mucosal tissues play critical roles in type 2 airway inflammatory conditions such as AR, eosinophilic chronic rhinosinusitis, and bronchial asthma. However, there have been a few reports about the role of blood ILC2s. The prevalence of blood ILC2s correlated positively with serum IL-13 levels in patients with HDM-induced AR, ${ }^{15}$ and blood ILC2 prevalence was increased at 4 hours after nasal challenge with cat allergen. ${ }^{17}$ Blood ILC2 prevalence was increased during the pollen season in patients with grass pollen-induced AR, and was decreased after subcutaneous allergen-specific immunotherapy. ${ }^{18}$ In the present study, there was a strong positive correlation between the prevalence of blood ILC2s and blood eosinophilia, and a weak positive correlation between the prevalence of blood ILC2s and serum IgE levels. In our representative severe case, resection of the mass lesion with tissue eosinophilia and systemic steroid treatment ameliorated clinical symptoms, with concomitant decreases of blood ILC2s, blood eosinophilia, and serum IgE levels. When the disease recurred, the prevalence of blood ILC2s and blood eosinophilia were increased. These results indicate that increased blood ILC2s with elevated ability to produce type 2 cytokines are associated with blood eosinophilia, elevated serum IgE levels, and clinical symptoms such as general itching, in patients with KD.

The most widely used treatments for KD are surgical resection of the mass lesions with tissue eosinophilia and systemic steroid therapy. ${ }^{2}$ Surgery helps to reach a definitive diagnosis. Systemic steroid therapy has a transient effect due to its anti-inflammatory effects, but how steroid therapy works in the pathogenesis of KD has not been investigated. In this study, dexamethasone inhibited the productions of IL- 5 and IL-13 by KD patient-derived ILC2s. In our representative case, systemic steroid treatment also suppressed the blood ILC2 prevalence, blood eosinophilia, and itching. Taken together, one of the reasons why systemic steroid therapy is effective in the treatment of KD might be due to corticosteroids inhibiting type 2 cytokine productions of blood ILC2s.

IL-5 is an essential cytokine that controls the development, maturation, and terminal differentiation of eosinophils, modulating the release of eosinophils from bone marrow. ${ }^{19}$ IL-4 and IL-13 recruit eosinophils from blood into tissues by inducing the production of adhesive ligands on the endothelium, such as CC chemokines and vascular cell adhesion molecule-1 (VCAM-1). ${ }^{19,20}$ In addition, eotaxin 1-3, macrophagederived chemokine (MDS), and thymus and activation-regulated chemokine (TARC) are involved in this process of eosinophil migration. ${ }^{19}$ Although the mechanisms of eosinophil activation and migration are not fully understood, IL-4, IL-5, and IL-13 are known to act together as key drivers in eosinophilic inflammation. Systematic reviews of the effects of anti-IL- $4 \mathrm{R} \alpha$ (both IL- 4 and IL-13 signaling), anti-IL-5, and anti-IL-5R $\alpha$ monoclonal antibodies show the clinical efficacy of these biological drugs in eosinophilic airway inflammation such as bronchial asthma and chronic rhinosinusitis with nasal polyps. ${ }^{19,21}$ The results of the present study suggest that blocking the activities of IL-4, IL-5, and IL-13 may have novel therapeutic potential for the treatment of patients with intractable KD.

Itching is a characteristic symptom of KD, and IL-4, IL-13, and IL-31 have been reported to stimulate sensory neurons and to induce itching. ${ }^{5,6}$ In the present study, serum IL-13 levels were significantly increased in patients with KD, and KD patient-derived blood ILC2s produced a large amount of IL-13 in response to $\mathrm{PGD}_{2}$ or $\mathrm{LTD}_{4}$. On the other hand, serum IL-31 levels were significantly decreased in patients with KD, and sorted blood ILC2s did not produce IL-31. These results indicate that ILC2-derived IL-13 may be responsible for itching in patients with KD. Serum IL-31 levels have been reported to be increased in patients with atopic dermatitis ${ }^{8}$ and bronchial asthma, ${ }^{22}$ and those elevated serum IL-31 levels have been shown to correlate positively with the severity of these diseases. ${ }^{8,23}$ In the present study, serum IL-31 levels also were increased in patients with AR. To our knowledge, $\mathrm{KD}$ is the only disease in which eosinophilic inflammation is combined with decreased serum IL-31 levels. IL-31 is produced primarily by Th2 cells, suggesting that the function of Th2 cells in KD will need to be evaluated in further studies.

In conclusion, increased blood ILC2s may be involved in blood eosinophilia and general itching through the production of IL-5 and IL-13 in patients with KD. Serum IL-13 levels also were increased, and ILC2- 
derived IL-13 may induce general itching in patients with KD. Although the etiology of KD remains unclear, regulation of blood ILC2s may have new therapeutic potential for the treatment of intractable KD. We hope that these findings will be confirmed and advanced by subsequent investigations.

\section{Conflicts of interest}

This research was supported, in part, by the Japan Society for the Promotion of Science (JSPS; Grant Numbers 17K16908 and 20K09754).

\section{References}

1. Chen H, Thompson LD, Aguilera NS, Abbondanzo SL. Kimura disease: a clinicopathologic study of 21 cases. Am J Surg Pathol.2004;28(4):505-513.

2. Kakehi E, Kotani K, Otsuka Y, Fukuyasu Y, Hashimoto Y, Sakurai S, et al. Kimura's disease: effects of age on clinical presentation. QJM 2020;113(5):336-345.

3. Kimura Y, Pawankar R, Aoki M, Niimi Y, Kawana S. Mast cells and T cells in Kimura's disease express increased levels of interleukin-4, interleukin-5, eotaxin and RANTES. Clin Exp Allergy.2002;32(12):1787-1793.

4. Katagiri K, Itami S, Hatano Y, Yamaguchi T, Takayasu S. In vivo expression of IL-4, IL-5, IL-13 and IFN-gamma mRNAs in peripheral blood mononuclear cells and effect of cyclosporine A in a patient with Kimura's disease. Br J Dermatol. 1997;137(6):972-977.

5. Oejen LK, Mack MR, Feng J, Whelan TM, Niu H, Guo CJ, et al. Sensory neurons co-opt classical immune signaling pathways to mediate chronic itch. Cell . 2017;171(1):217-228.

6. Weidinger S, Beck LA, Bieber T, Kabashima K, Irvine AD. Atopic dermatitis. Nat Rev Dis Primers . $2018 ; 4(1): 1$.

7. Stott B, Lavender P, Lehmann S, Pennino D, Durham S, Schmidt-Weber CB. Human IL-31 is induced by IL-4 and promotes TH2-driven inflammation. J Allergy Clin Immunol . 2013;132(2):446-454.e5.

8. Nakashima C, Otsuka A, Kabashima K. Interleukin-31 and interleukin-31 receptor: New therapeutic targets for atopic dermatitis. Exp Dermatol. 2018;27(4):327-331.

9. Schleimer RP. Immunopathogenesis of chronic rhinosinusitis and nasal polyposis. Annu Rev Pathol. 2017;12:331-357.

10. Tojima I, Matsumoto K, Kikuoka H, Hara S, Yamamoto S, Shimizu S, et al. Evidence for the induction of Th2 inflammation by group 2 innate lymphoid cells in response to prostaglandin D2 and cysteinyl leukotrienes in allergic rhinisits. Allergy . 2019;74(12):2417-2426.

11. Kabata H, Moro K, Koyasu S. The group 2 innate lymphoid cell (ILC2) regulatory network and its underlying mechanisms. Immunol Rev . 2018:286(1):37-52.

12. Miyata J, Fukunaga K, Kawashima Y, Ohara O, Arita M. Cysteinyl leukotriene metabolism of human eosinophils in allergic disease. Allergol Int . 2020;69(1):28-34.

13. Luna-Gomes T, Magalhães KG, Mesquita-Santos FP, Bakker-Abreu I, Samico RF, Molinaro R, et al. Eosinophils as a novel cell source of prostaglandin D2: autocrine role in allergic inflammation. $J$ Immunol . 2011;187(12):6518-6526.

14. Bousquet J, Khaltaev N, Cruz AA, Denburg J, Fokkens WJ, Togias A, et al. Allergic Rhinitis and its Impact on Asthma (ARIA) 2008 update (in collaboration with the World Health Organization, GA(2)LEN and AllerGen). Allergy . 2008;63 Suppl 86:8-160.

15. Zhong H, Fan XL, Yu QN, Qin ZL, Chen D, Xu R, et al. Increased innate type 2 immune response in house dust mite-allergic patients with allergic rhinitis. Clin Immunol . 2017;183:293-299.

16. Tojima I, Kouzaki H, Shimizu S, Ogawa T, Arikata M, Kita H, et al. Group 2 innate lymphoid cells are increased in nasal polyps in patients with eosinophilic chronic rhinosinusitis. Clin Immunol . 2016;170:1-8.

17. Doherty TA, Scott D, Walford HH, Khorram N, Lund S, Baum R, et al. Allergen challenge in allergic rhinitis rapidly induces increased peripheral blood type 2 innate lymphoid cells that express CD84.J Allergy Clin Immunol. 2014;133(4):1203-1205.

18. Lao-Araya M, Steveling E, Scadding GW, Durham SR, Shamji MH. Seasonal increases in peripheral 
innate lymphoid type 2 cells are inhibited by subcutaneous grass pollen immunotherapy. J Allergy Clin Immunol. 2014;134(5):1193-1195.e4.

19. Akdis CA, Arkwright PD, Brüggen MC, et al. Type 2 imunity in the skin and lungs. Allergy . 2020;75(7):1582-1605.

20. Nagata M, Nakagome K, Soma T. Mechanisms of eosinophilic inflammation.Asia Pac Allergy . 2020;10(2):e 14.

21. Oykhman P, Paramo FA, Bounsquet J, et al. Comparative efficacy of monoclonal antibodies and aspirin desensitization for chronic rhinosinusitis with nasal polyposis: A systematic review and network metaanalysis. J Allergy Clin Immunol.2021:S0091-6749(21)01393-2.

22. Lei Z, Liu G, Huang Q, Lv M, Zu R, Zhang GM, et al. SCF and IL-31 rather than IL-17 and BAFF are potential indicators in patients with allergic asthma. Allergy . 2008;63(3):327-332.

23. Lai T, Wu D, Li W, Chen M, Yi Z, Huang D, et al. Interleukin-31 expression and relation to disease severity in human asthma. Sci Rep . 2016;6:22835.

Table 1

Subject characteristics

\begin{tabular}{|c|c|c|c|}
\hline & $\begin{array}{l}\text { Control subjects }(\mathrm{n}= \\
13)\end{array}$ & $\begin{array}{l}\text { HDM-induced AR }(\mathrm{n}= \\
16)\end{array}$ & $\mathrm{KD}(\mathrm{n}=4)$ \\
\hline Age $(\mathrm{y})$, mean \pm SEM & $38.8 \pm 3.0$ & $30.8 \pm 2.2$ & $34.3 \pm 9.1$ \\
\hline $\operatorname{Sex}(M / F)$ & $8 / 5$ & $11 / 5$ & $4 / 0$ \\
\hline $\begin{array}{l}\text { Blood eosinophil (\%), } \\
\text { mean } \pm \text { SEM }\end{array}$ & $2.1 \pm 0.5$ & $3.7 \pm 0.5$ & $14.2 \pm 2.8^{* *}+$ \\
\hline $\begin{array}{l}\text { Serum total IgE } \\
(\mathrm{IU} / \mathrm{mL}), \text { mean }+- \\
\text { SEM }\end{array}$ & $56 \pm 30$ & $625 \pm 475^{*}$ & $1652 \pm 181^{* *}$ \\
\hline $\begin{array}{l}\text { HDM sIgE (class), mean } \\
\pm \text { SEM }\end{array}$ & 0 & $3.1 \pm 0.4^{* *}$ & $0.3 \pm 0.3+$ \\
\hline Itching $(\%)$ & 0 & 25 & $100^{* * *}+$ \\
\hline
\end{tabular}

$H D M$, House dust mite. $A R$, Allergic rhinitis. $K D$, Kimura disease.

${ }^{*} P<0.05,{ }^{* *} P<0.01,{ }^{* * *} P<0.001$, versus control subjects.

$+P<0.05$, versus patients with HDM-induced AR.

Figure legends

Figure 1. The prevalence of blood ILC2s is increased in patients with Kimura disease (KD). A , Representative flow cytometry scattergrams for group 2 innate lymphoid cells (ILC2s, red box) in peripheral blood mononuclear cells (PBMCs) from a control subject, a patient with house dust mite (HDM) -induced allergic rhinitis (AR), and two patients with Kimura disease (KD). B , Blood ILC2 prevalence in control subjects, patients with HDM-induced AR, and patients with $\mathrm{KD} .{ }^{*} P<0.05 ;{ }^{*} P<0.01$, evaluated using the two-tailed Kruskal-Wallis test with Dunn'spost hoc test. C and D , Correlation between blood ILC2 prevalence and the percentage of blood eosinophils $(\mathbf{C})$, and serum IgE levels (D) in all study subjects.

Figure 2. Serum concentrations of IL-13 (A ), IL-5 (B ), IL-4 (C ), and IL-31 (D ) in control subjects, patients with HDM-induced AR, and patients with KD. Data are shown as the mean \pm SEM. ${ }^{*} P<0.05$; ${ }^{* *} P<0.01 ;{ }^{* * *} P<0.001$. Comparisons between groups were evaluated using the two-tailed Kruskal-Wallis test with Dunn's post hoc test.

Figure 3. Correlation between blood ILC2 prevalence and serum concentrations of IL-13 (A), IL-5 (B), IL-4 (C), or IL-31 (D). 
Figure 4. The production of IL-4, IL-5, and IL-13 from blood ILC2s. A , Sorted blood ILC2s from patients with KD were cultured for 7 days in medium containing IL-2 $(50 \mathrm{ng} / \mathrm{mL})$ alone or in IL-2-containing medium supplemented with IL-33 $(10 \mathrm{ng} / \mathrm{mL})$, prostaglandin $(\mathrm{PG}) \mathrm{D}_{2}(1 \mu \mathrm{M})$, or leukotriene (LT) $\mathrm{D}_{4}(50 \mathrm{nM})$. Data are shown as the mean \pm SEM. ${ }^{*} P<0.05 ;{ }^{* *} P<0.01 ;{ }^{* * *} P<0.001$, versus medium with IL-2 only. ${ }^{\#} P$ $<0.05$; \#\# $P<0.01$; \#\#\# $P<0.001$, versus IL-33. Comparisons between groups were evaluated using the twotailed Mann-Whitney $U$ test. B , Sorted blood ILC2s from control subjects, patients with HDM-induced $\mathrm{AR}$, and patients with KD were cultured for 7 days in medium containing IL-2 (50 ng/mL) alone or in IL2-containing medium supplemented with $\mathrm{PGD}_{2}$ or $\mathrm{LTD}_{4}$. Data are shown as median values (horizontal lines ), interquartile ranges (boxes), and 1.5 interquartile range (whiskers ). ${ }^{*} P<0.05 ;{ }^{* *} P<0.01 ;{ }^{* * *} P<0.001$. C , IL-5 and IL-13 productions from PBMC-derived ILC2s from patients with KD. Proliferated ILC2s were incubated with or without $\mathrm{PGD}_{2}(1 \mu \mathrm{M})$ or $\mathrm{LTD}_{4}(50 \mathrm{nM})$, with or without dexamethasone (Dex, $10^{-8}$ to $\left.10^{-6} \mathrm{M}\right)$ in the presence of IL-2 $(50 \mathrm{ng} / \mathrm{mL})$ for $12 \mathrm{~h}$. Data are shown as the mean \pm SEM. ${ }^{*} P<0.05 ;{ }^{* *} P$ $<0.01$.

Figure 5. Changes in the prevalence of blood ILC2s, blood eosinophilia, clinical symptoms, MRI findings, and photographs in a representative severe case before and after surgery and steroid therapy. $\mathbf{A}$, Changes in blood ILC2 prevalence and the percentage of blood eosinophils. PSL, prednisolone; d, day; w, week; $\mathrm{m}$, month; y, year.B , Changes in blood ILC2 prevalence and the visual analog scale (VAS) scores of general itching and distress. C , Changes in axial and coronal MRI (T2-weighted images) showing a mass lesion in the right parotid area (red arrows). D , Changes in swelling of right parotid area (red arrows).

\section{Hosted file}

Figure 1_Tojima et al.docx available at https://authorea.com/users/334207/articles/552281increased-blood-group-2-innate-lymphoid-cells-are-involved-in-blood-eosinophilia-anditching-in-kimura-disease

\section{Hosted file}

Figure 2_Tojima et al.docx available at https://authorea.com/users/334207/articles/552281increased-blood-group-2-innate-lymphoid-cells-are-involved-in-blood-eosinophilia-anditching-in-kimura-disease

\section{Hosted file}

Figure 3_Tojima et al.docx available at https://authorea.com/users/334207/articles/552281increased-blood-group-2-innate-lymphoid-cells-are-involved-in-blood-eosinophilia-anditching-in-kimura-disease

\section{Hosted file}

Figure 4_Tojima et al.docx available at https://authorea.com/users/334207/articles/552281increased-blood-group-2-innate-lymphoid-cells-are-involved-in-blood-eosinophilia-anditching-in-kimura-disease

\section{Hosted file}

Figure 5_Tojima et al.docx available at https://authorea.com/users/334207/articles/552281increased-blood-group-2-innate-lymphoid-cells-are-involved-in-blood-eosinophilia-anditching-in-kimura-disease 